\title{
Micellar Liquid Chromatography: Recent Advances and Applications
}

\author{
Samuel Carda-Broch, ${ }^{1}$ Josep Esteve-Romero, ${ }^{1}$ Maria Rambla-Alegre, ${ }^{2}$ \\ Maria Jose Ruiz-Angel, ${ }^{3}$ Alain Berthod, ${ }^{4}$ and Devasish Bose ${ }^{5}$ \\ ${ }^{1}$ Physical and Analytical Chemistry Department, University Jaume I, 12071 Castellon, Spain
2 Organic Chemistry Department, Ghent University, 9000 Ghent, Belgium
${ }^{3}$ Analytical Chemistry Department, University of Valencia, 46100 Burjasot, Spain
${ }^{4}$ Laboratoire des Sciences Analytiques, Université de Lyon, CNRS, 69622 Villeurbanne, France
${ }^{5}$ Department of Criminology and Forensic Science, Dr. H.S.Gour University, Sagar, India
}

Correspondence should be addressed to Samuel Carda-Broch, samuel.carda@qfa.uji.es

Received 5 April 2012; Accepted 5 April 2012

Copyright ( 2012 Samuel Carda-Broch et al. This is an open access article distributed under the Creative Commons Attribution License, which permits unrestricted use, distribution, and reproduction in any medium, provided the original work is properly cited.

Micellar liquid chromatography (MLC) is a reversed-phase liquid chromatographic (RPLC) mode with a mobile phase consisting in an aqueous solution of surfactant above its critical micellar concentration (CMC). The idea of using pure micellar solutions as mobile phases in RPLC is very attractive given their lower cost, less toxicity, and poorer environmental impact. In practice, however, the addition of a small amount of organic solvent to the micellar solution is needed to achieve retention in practical time windows and to improve peak efficiency and resolution. MLC also provides a solution to the direct injection of real samples (physiological or food) by solubilising proteins. The possibility of directly injecting samples into the chromatograph simplifies and expedites treatment, which confers analytical procedures of greater accuracy and a lower cost. Fundamental studies into MLC have served to develop the technique and to establish its theoretical basis, without which its later use in diverse applications would be impossible. In this special issue on MLC, we have invited a few papers that address such issues.

A paper by M. Rambla-Alegre describes the basics of MLC, highlighting the particularities of this technique over the RPLC. The partitioning equilibria are described in detail. It also introduces the term of submicellar liquid chromatography. Another paper by the same author lies on the description of the retention in MLC. The complexity of the mixtures of compounds studied and the relevant modification of their chromatographic behavior when changing the mobile phase composition requires the use of computerassisted simulations in MLC to follow the modifications in the chromatograms in detail. These simulations can be done with sound reliability thanks to the use of chemometrics tools. The most frequently used empirical and mechanistic models that describe the retention behavior of compounds are revised. The modeling of peak shape and the strategies to measure the peak resolution are also discussed.

A paper by $\mathrm{N}$. Memon et al. studies the selectivity of a non ionic surfactant (Brij-35) in MLC separation of positional isomers. The effect of surfactant and organic solvent concentration on the separation of some selected isomers is studied and evaluated in terms of Linear Solvation energy Relationship (LSER). Non ionic MLC offers different mode of interaction than hydro-organic or ionic micellar liquid chromatography. Besides basicity, dipolarizability and excess molar refraction are responsible for fine tuning of separation. This new face of non-ionic MLC opens field for many applications in separation of positional isomers.

In the paper by A. U. Kulikov an MLC method was developed and validated according to $\mathrm{ICH}$ guidelines for the determination of sesquiterpenic acids in root and rhizome extract from Valeriana officinalis and valerian dry hydroalcoholic extract. The proponed method does not require gradient elution which is widely used in reversedphase HPLC methods. This is one of the main advantages of MLC that allows separating compounds with different 
hydrophobicity in a single run without the gradient elution. This is an application that denotes the usefulness of this technique.

In the paper by M.-L. Chin-Chen another applied work that determines the levels of the biogenic amine spermine in anchovy sauce after derivatization with 3,5-dinitrobenzoyl chloride is introduced. The suggested methodology was found useful in routine analysis of spermine in fish sauce samples. This methodology was validated in terms of linearity, sensitivity, limits of detection and quantification, accuracy, precision and recovery, following the FDA guidelines. Direct injection of samples (after filtration) was used, thus avoiding any tedious extraction and purification step. This is another interesting advantage of the MLC technique.

\author{
Samuel Carda-Broch \\ Josep Esteve-Romero \\ Maria Rambla-Alegre \\ Maria Jose Ruiz-Angel \\ Alain Berthod \\ Devasish Bose
}




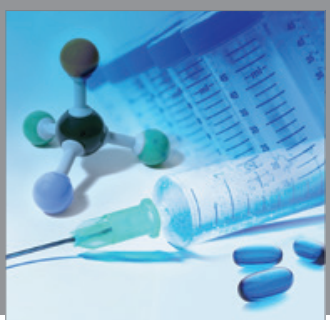

International Journal of

Medicinal Chemistry

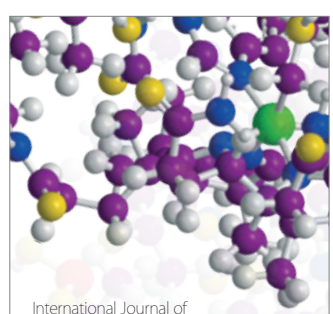

Carbohydrate Chemistry

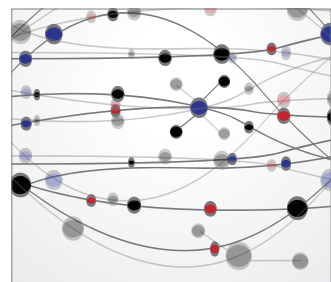

The Scientific World Journal
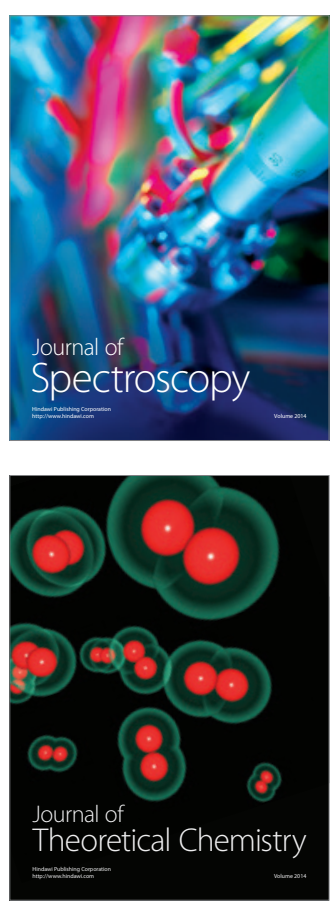
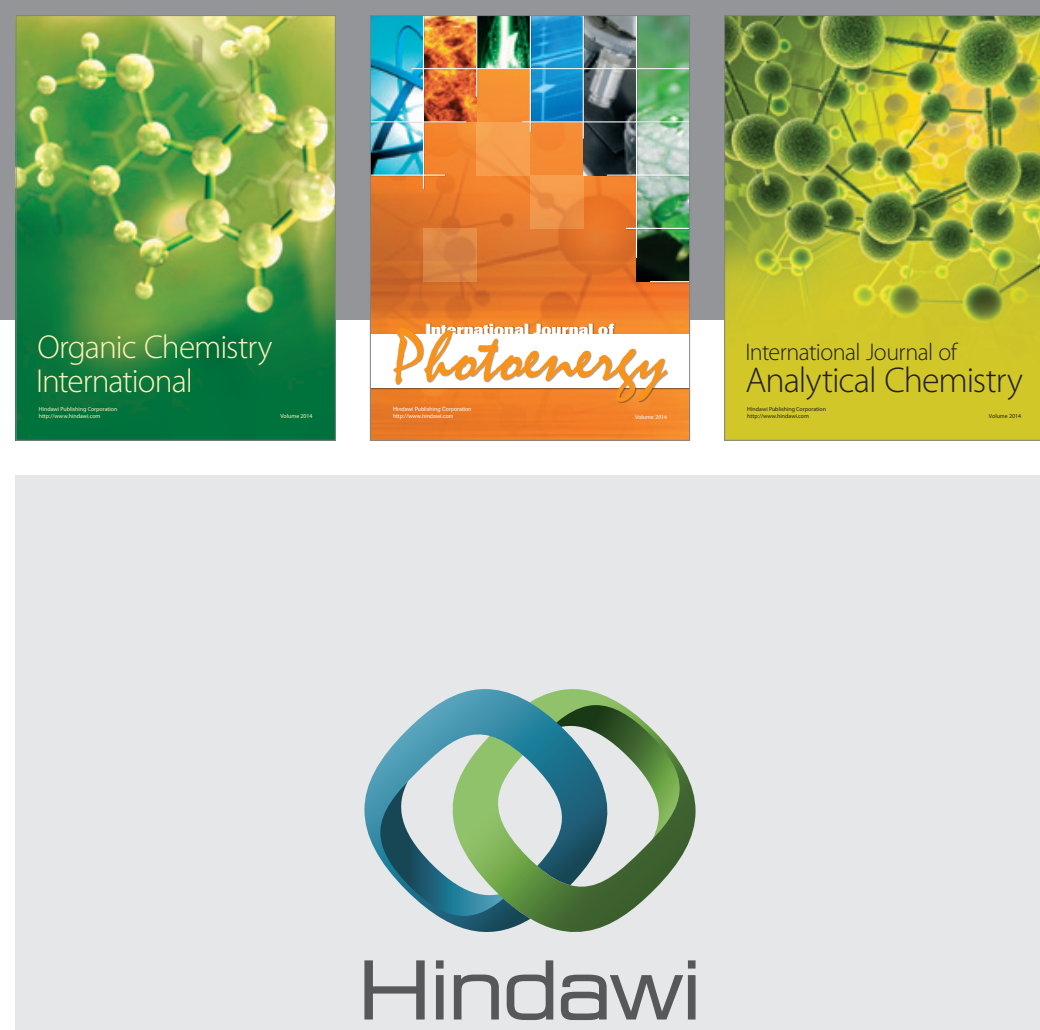

Submit your manuscripts at

http://www.hindawi.com
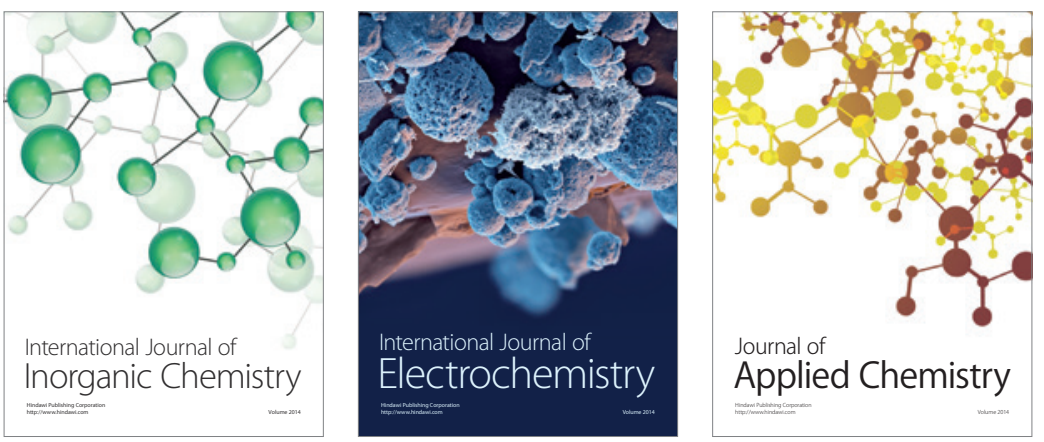

Journal of

Applied Chemistry
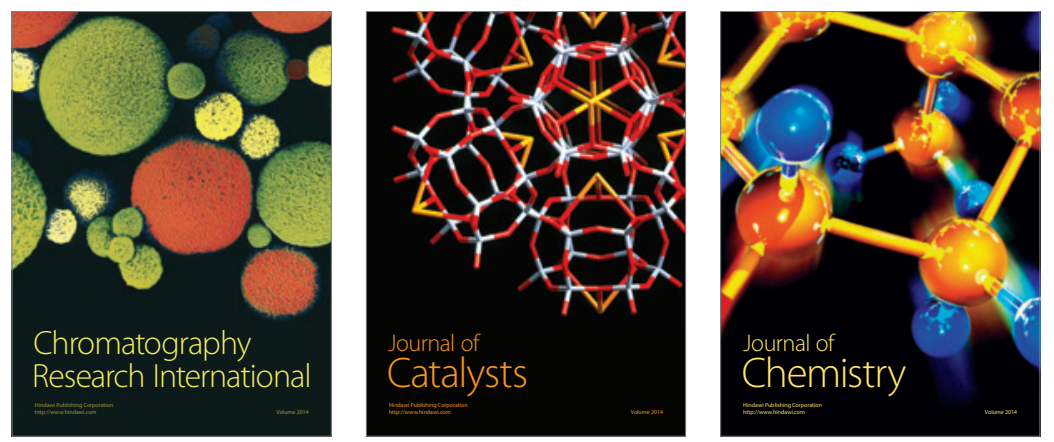
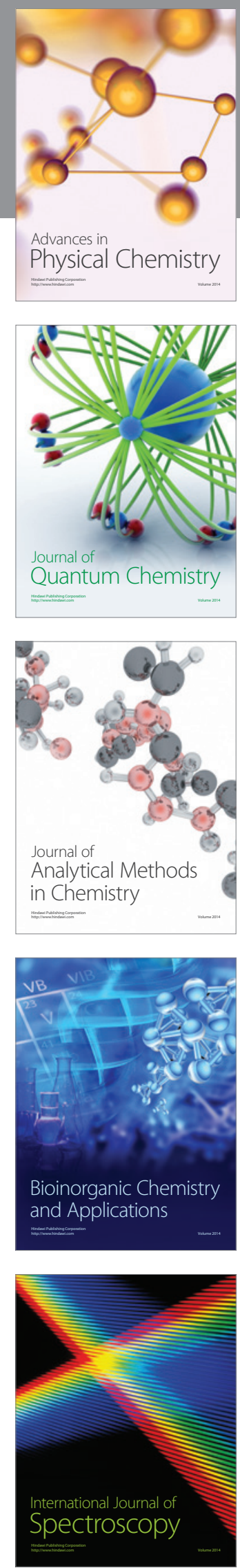